\title{
Ki67 and progesterone receptor status predicts sensitivity to palbociclib: a real-world study
}

\author{
Xiying Shao ${ }^{1,2}$, Yabing Zheng ${ }^{1,2}$, Wenming Cao ${ }^{1,2}$, Xiabo Shen $^{1,2} \wedge$, Guangliang Li $^{1,2}$, Junqing Chen ${ }^{1,2}$, \\ Yuan Huang ${ }^{1,2}$, Ping Huang ${ }^{1,2}$, Lei Shi ${ }^{1,2}$, Weiwu Ye ${ }^{1,2}$, Weibin Zou ${ }^{1,2}$, Caijin Lou ${ }^{1,2}$, Lei Lei ${ }^{1,2}$, Jian Huang ${ }^{1,2}$, \\ Zhanhong Chen ${ }^{1,2}$, Xiaojia Wang ${ }^{1,2}$ \\ ${ }^{1}$ Department of Medical Oncology (Breast Cancer), Cancer Hospital of the University of Chinese Academy of Sciences/Zhejiang Cancer Hospital, \\ Hangzhou, China; ${ }^{2}$ Institute of Cancer and Basic Medicine (ICBM), Chinese Academy of Sciences, Hangzhou, China \\ Contributions: (I) Conception and design: X Wang, X Shao; (II) Administrative support: Z Chen, Y Zheng; (III) Provision of study materials or \\ patients: Wenming Cao, W Ye, W Zou, C Lou; (IV) Collection and assembly of data: X Shen, G Li, J Chen, Y Huang, J Huang; (V) Data analysis \\ and interpretation: L Lei, L Shi, P Huang; (VI) Manuscript writing: All authors; (VII) Final approval of manuscript: All authors. \\ Correspondence to: Xiaojia Wang; Zhanhong Chen. Department of Medical Oncology (Breast Cancer), Cancer Hospital of the University of Chinese \\ Academy of Sciences/Zhejiang Cancer Hospital, Hangzhou, China. Email: wxiaojia0803@163.com; czred@sina.com.
}

Background: Palbociclib combined with endocrine therapy has been approved as a front-line treatment for hormone receptor positive (HR+), human epidermal growth factor receptor 2 negative (HER2-) advanced breast cancer (ABC). A key challenge remains to uncover biomarkers to identify those patients who may benefit from palbociclib treatment.

Methods: We retrospectively analyzed the values of Ki67 and progesterone receptor (PR) as detected by immunohistochemistry in $81 \mathrm{ABC}$ patients with palbociclib and hormone therapy treatment, and evaluated the impact on progression-free survival (PFS).

Results: In the total population, women with Ki67 $\geq 14 \%$ had marginally significantly shorter PFS than those with Ki67 <14\% (P=0.062). Patients with Ki67 $\geq 30 \%$ had significantly shorter PFS than those with Ki67 $<30 \%$ ( $\mathrm{P}=0.048$ ). Meanwhile, $\mathrm{PR} \geq 20 \%$ was associated with longer PFS. Moreover, the change of Ki67 or PR from primary tissue to metastatic lesions was related to PFS. As for the hormone therapy subgroup, there were significant associations between Ki67 and PR levels and PFS in the aromatase inhibitors (AIs) subgroup. Patients with Ki67 $\geq 14 \%$ or Ki67 $\geq 30 \%$ had shorter PFS than those with Ki67 $<14 \%$ or Ki67 $<30 \%$, respectively $(\mathrm{P}=0.024, \mathrm{P}<0.001)$. Additionally, the change of Ki67 or PR from primary tissue to metastatic lesions was related to PFS. When both Ki67 and PR were considered, there were significant differences between the different cohorts. Compared with patients with Ki67 $\geq 14 \%$ and PR $<20 \%$, those with Ki67 $<14 \%$ and PR $\geq 20 \%$ had significantly longer PFS. In addition, patients with Ki67 $<30 \%$ and PR $\geq 20 \%$ had significantly longer PFS than those with Ki67 $\geq 30 \%$ and PR $<20 \%$. Furthermore, in the AI cohort, patients with Ki67 $<14 \%$ and PR $\geq 20 \%$ had significantly longer PFS than those with Ki67 $\geq 14 \%$ and PR $<20 \%$. Women with Ki67 $<30 \%$ and PR $\geq 20 \%$ had significantly longer PFS than those with Ki67 $\geq 30 \%$ and $\mathrm{PR}<20 \%$.

Conclusions: The present study indicates that both Ki67 and PR have great impacts on palbociclib and hormone therapy and may contribute to selecting more effective partners for palbociclib.

Keywords: Ki67; progesterone receptor; palbociclib

Submitted Feb 03, 2021. Accepted for publication Apr 17, 2021.

doi: $10.21037 / \mathrm{atm}-21-1340$

View this article at: http://dx.doi.org/10.21037/atm-21-1340

^ ORCID: 0000-0002-1643-3842. 


\section{Introduction}

Hormone therapy is the primary treatment in patients with hormone receptor positive $(\mathrm{HR}+)$ and human epidermal growth factor receptor 2 negative (HER2-) advanced breast cancer (ABC) (1). However, resistance through diverse mechanisms is eventually inevitable, leading to treatment failure and disease progression for patients (2-4). Immense progress has been reached in HR+/HER2- ABC since the approval of cyclin-dependent kinase (CDK)4/6 inhibitor (CDK4/6i), palbociclib, in 2015, according to data from the PALOMA-1 trial $(5,6)$.

CDK4/6i works by inhibiting the interaction of cyclins 4 and 6 with cyclin $\mathrm{D}$, thus arresting the hyperphosphorylation of retinoblastoma $(\mathrm{Rb})$ and blocking progression from G1 to $\mathrm{S}$ phase of the cell cycle, thereby inhibiting transcription in turn $(7,8)$. Many phase III randomized trials have demonstrated the effectiveness of CDK4/6i in both first-line (9-12) and endocrine-resistant settings (12-14). Palbociclib, a first-in-class CDK4/6 inhibitor, was approved for the treatment of HR+/HER2ABC. The most common adverse effects of palbociclib are neutropenia, leukopenia, and fatigue (10). In the PALOMA-3 and PALOMA-2 trials, grade 3-4 neutropenia occurred in $62.0 \%$ and $66.4 \%$ of patients, respectively, while febrile neutropenia occurred in only $1.8 \%$ and $0.6 \%$ of patients, respectively $(11,12,15)$.

Although evidence based on randomized trials is the gold standard (16), its translation to clinical practice can be problematic. In the palbociclib randomized trials, most of patients had relapsed after no more than one line of chemotherapy or the patients were even treatment naïve, while the majority of patients randomized to the placebo cohorts never had palbociclib at the late line. Furthermore, the data from real-world studies have revealed an inferior benefit of CDK4/6i in heavily pretreated patients when compared to the data reported in prospective trials $(17,18)$, which suggest that patient selection may be a determinant cause behind outcomes of the clinical trials. Additionally, adherence to dose modification guidelines in clinical trials is presumably higher than that in clinical practice, with the prognostic implications of this difference being largely unexplored. Finally, no robust predictive or prognostic biomarkers which may guide clinical medication have been explored in the randomized trials of palbociclib (19).

The PALOMA-3 trial found that high progesterone receptor (PR) expression (performed by $\mathrm{H}$-score) was related to superior benefit, independent of the treatment cohort (20). Also, retinoblastoma protein ( $\mathrm{Rb}$ )-negative cases have been shown to have high Ki67 levels (21). In addition, Palleschi et al. (22) revealed that the progressionfree survival (PFS) of patients with CDK4/6i and endocrine therapy was inversely related to Ki67 expression, but not to PR. Thus, studies concerning the predictive significance of Ki67 and PR for CDK4/6i are rare and controversial. In this retrospective study, we used immunohistochemistry (IHC) to evaluate the prognostic role of the values of Ki67 (proliferative index) and PR, with PFS being considered the main measure.

We present the following article in accordance with the REMARK reporting checklist (available at http://dx.doi. org/10.21037/atm-21-1340).

\section{Methods}

\section{Study design}

This was a retrospective cohort study of metastatic or locally advanced and unresectable HR+/HER2- BC patients initiating treatment with palbociclib in combination with hormone therapy in the 2-year interval following approval of palbociclib in China. A total of 150 patients were identified in the Cancer Hospital of the University of Chinese Academy of Sciences/Zhejiang Cancer Hospital. This retrospective study was conducted in line with accepted ethical standards and was approved by the Medical Scientific Committee of Cancer Hospital of the University of Chinese Academy of Sciences/Zhejiang Cancer Hospital (No. IRB-2021-19). All procedures performed in this study involving human participants were in accordance with the Declaration of Helsinki (as revised in 2013). Individual consent for this retrospective analysis was waived. Only 81 patients, those who received rebiopsy of metastatic lesions and had Ki67 results, were enrolled in the present analysis. Data were collected through the institution's electronic medical record database. Patients who had undergone least one cycle of palbociclib between 10 August 2018 and 31 August 2020 and who were aged 18 years or older at initiation of palbociclib were included. Patients were excluded from the study if any of the following conditions were present: (I) non-breast cancer; (II) palbociclib treatment as part of a clinical trial or prescription prior to 10 August 2018; or (III) no record of concomitant treatment with aromatase inhibitors (AIs) or fulvestrant; (IV) no results of Ki67 status of metastasis.

The primary outcomes in the study were the predictive 
significance of Ki67 and PR expression for hormone therapy plus CDK4/6i. The second aims were the predictive significance of Ki67 and PR expression for AIs plus CDK4/6i or fulvestrant plus CDK4/6i. The PFS was recorded as the duration from the beginning of treatment to disease progression or death, whichever happened first.

Palbociclib was considered as first-line therapy (LOT1) if it was given in the setting of no prior systemic treatment for $\mathrm{ABC}$ or at least 1 year from the completion of adjuvant endocrine therapy. Palbociclib was considered as secondline therapy (LOT2) if given postprogression on a firstline treatment for $\mathrm{ABC}$ or on relapse at or within 1 year from the end of adjuvant hormone therapy. Palbociclib was defined as third-line therapy (LOT3) if received beyond second-line therapy for $\mathrm{ABC}$.

\section{Biomarker detection}

Estrogen receptor (ER), PR, and Ki67 were evaluated by IHC, in line with European Quality Assurance guidelines. HER2 evaluation was performed by HercepTest (Dako Corporation) or fluorescence in situ hybridization (FISH) using the dual color FISH-PathVysion kit (Kanglu) at Zhejiang Cancer Hospital. Immunostaining was conducted with the Ventana Benchmark XT system and the Ultraview DAB Detection Kit (Ventana Medical Systems). Confirm anti-ER (Clone SP1, Dako), confirm anti-PR (Clone PgR636, Dako), and Ki67 (Clone Mib-1, Dako,) antibodies were used. Sections were automatically counterstained with hematoxylin II (Ventana Medical Systems) for 16 minutes. External negative and positive controls were taken for each test. Positivity was examined and semiquantitatively quantified as the percentage between immunopositive neoplastic cells and the whole neoplastic cells. HR and HER2 status were defined by the American Society of Clinical Oncology (ASCO)/College of American Pathologists guidelines (23,24). In particular, the cutoff for ER positivity and PR positivity was set at $\geq 1 \%$ immunoreactive cells. Ki67 and PR were analyzed both as continuous and dichotomized variables; i.e., low $(<14 \%)$ $v s$. high $(\geq 14 \%)$ for Ki67, low $(<20 \%)$ vs. high $(\geq 20 \%)$ for PR in accordance with the St. Gallen guidelines (25) and low $(<30 \%)$ vs. high $(\geq 30 \%)$ for Ki67 according to the Guidelines of the Chinese Society of Clinical Oncology (CSCO) on Diagnosis and Treatment of Breast Cancer 2020.

HER2 status was conducted using the HercepTest, which measures the percentage of immunoreactive tumor cells according to the intensity and completeness of membrane staining and with the $0-3+$ recommended criterion. Cases scored as $3+$ were defined as HER $2+$. In cases with a score of $2+$, a FISH test was conducted. HER2 status was considered positive if the HER2 gene-chromosome 17 centromere ratio was $\geq 2$ or if the average HER2 gene copy number/cell was $\geq 6$ according to FISH.

\section{Statistical analyses}

The follow-up period was defined as the time from initiation of one regimen to the earliest disease progression, death, or last medical record. The median follow-up period was evaluated by the reverse Kaplan-Meier method. The Kaplan-Meier method was performed to estimate PFS, and survival curves were compared using the log-rank test (26). Results were considered statistically significant with a $\mathrm{P}$ value $<0.05$. Analyses were conducted with SPSS (version 22.0, IBM Corp.) and the GraphPad Prism software (version 5, GraphPad Software Inc.).

\section{Results}

\section{Patient characteristics}

Between August 2018 and August 2020, 150 patients received palbociclib treatment at our institution. A total of 81 patients with Ki67 status in metastatic lesions were included. The median follow-up time was 12 months (range, 2.3-27.5 months). The patients' demographic and clinicopathologic characteristics and previous treatment history at the adjuvant and metastatic settings are summarized in Table 1. Follow-up for outcomes was completed on November 31, 2020. The median age was 54 years (range, $47-59$ years); $76.5 \%(n=62)$ of patients were aged $<60$ years and $23.5 \%(n=19)$ were aged $\geq 60$ years. In addition, $22.2 \%(\mathrm{n}=18)$ were premenopausal and $77.8 \%(n=63)$ were postmenopausal. All patients received the recommended dose of palbociclib $(125 \mathrm{mg}$ daily for 21 consecutive days, followed by 7 days off treatment) at the beginning; $51.9 \%(\mathrm{n}=42)$ patients received a combination of palbociclib and an AI, and 48.1\% ( $\mathrm{n}=39)$ received the treatment with fulvestrant (Table 2). Regarding line of therapy, $19.8 \%(\mathrm{n}=16)$ were in LOT1, $50.6 \%(\mathrm{n}=41)$ were in LOT2, and $29.6 \%(n=24)$ were in the subsequentline settings (Table 2). Only 9 patients $(11.1 \%)$ were de novo metastatic disease, while 72 patients $(88.9 \%)$ had recurrent metastatic breast cancer (Table 1). 
Page 4 of 12

Table 1 Baseline characteristics of 81 recurrence and metastasis breast cancer patients

\begin{tabular}{|c|c|}
\hline Clinical characteristics & Cases, n (\%) \\
\hline \multicolumn{2}{|l|}{ Age (year) } \\
\hline$<60$ & $62(76.5)$ \\
\hline$\geq 60$ & $19(23.5)$ \\
\hline Median [IQR] & 54.0 [47-59] \\
\hline \multicolumn{2}{|l|}{ Menopausal Status } \\
\hline Premenopausal & $18(22.2)$ \\
\hline Postmenopausal & $63(77.8)$ \\
\hline \multicolumn{2}{|l|}{ Pathological type (primary) } \\
\hline Invasive ductal carcinoma & $61(75.3)$ \\
\hline Invasive lobular carcinoma & $3(3.7)$ \\
\hline Others & $8(9.9)$ \\
\hline Unknown & $9(11.1)$ \\
\hline \multicolumn{2}{|l|}{ Grade $^{\#}$} \\
\hline 1 & $2(2.5)$ \\
\hline 2 & $37(33.3)$ \\
\hline 3 & $15(18.5)$ \\
\hline Unknown & $37(45.7)$ \\
\hline \multicolumn{2}{|l|}{ Tumor size ${ }^{\#}$} \\
\hline $\mathrm{T}<2 \mathrm{~cm}$ & $13(16.0)$ \\
\hline $\mathrm{T} \geq 2 \mathrm{~cm}$ & $43(53.1)$ \\
\hline Unknown & $25(30.9)$ \\
\hline \multicolumn{2}{|l|}{ N stage ${ }^{\#}$} \\
\hline No & $16(19.8)$ \\
\hline N1 & $20(24.7)$ \\
\hline N2 & $21(25.9)$ \\
\hline N3 & $10(12.3)$ \\
\hline Unknown & $14(17.3)$ \\
\hline \multicolumn{2}{|l|}{ PR status ${ }^{\#}$} \\
\hline$<20 \%$ & $30(37.0)$ \\
\hline$\geq 20 \%$ & $44(54.3)$ \\
\hline Unknown & $7(8.7)$ \\
\hline
\end{tabular}

Table 1 (continued)
Shao et al. Ki67 and PR status predicts sensitivity to palbociclib

Table 1 (continued)

\begin{tabular}{|c|c|}
\hline Clinical characteristics & Cases, n (\%) \\
\hline \multicolumn{2}{|l|}{$\mathrm{Ki} 67^{\#}$} \\
\hline $0-13 \%$ & $12(14.9)$ \\
\hline $14-29 \%$ & $21(25.9)$ \\
\hline$\geq 30 \%$ & $21(25.9)$ \\
\hline Unknown & 27 (33.3) \\
\hline \multicolumn{2}{|l|}{ Enrollment classification } \\
\hline De novo metastatic disease & $9(11.1)$ \\
\hline Recurrent disease & $72(88.9)$ \\
\hline \multicolumn{2}{|l|}{ ER status* } \\
\hline$(-)$ & $2(2.5)$ \\
\hline$(+)$ & $79(97.5)$ \\
\hline \multicolumn{2}{|l|}{ PR status* } \\
\hline$<20 \%$ & $57(70.4)$ \\
\hline$\geq 20 \%$ & $24(29.6)$ \\
\hline \multicolumn{2}{|l|}{$\mathrm{Ki} 67^{*}$} \\
\hline $0-13 \%$ & $18(22.2)$ \\
\hline $14-29 \%$ & $26(32.1)$ \\
\hline$\geq 30 \%$ & $37(45.7)$ \\
\hline \multicolumn{2}{|l|}{ Metastatic sites } \\
\hline Bone or soft tissue & $47(58.0)$ \\
\hline Visceral disease & $70(86.4)$ \\
\hline Lungs or pleura & $46(56.8)$ \\
\hline Liver & $35(43.2)$ \\
\hline Brain & $5(6.2)$ \\
\hline \multicolumn{2}{|l|}{ No. of organ metastases } \\
\hline$\leq 1$ & $34(42.0)$ \\
\hline$\geq 2$ & $47(58.0)$ \\
\hline
\end{tabular}

", primary tumor; *, metastases. ER, estrogen receptor; PR, progesterone receptor. IQR, inter-quartile range.

\section{Efficacy of palbociclib treatment according to different} Ki67 and PR status

Data on Ki67 and PR status for metastatic lesions were available in all of 81 patients (Table 1), while data for Ki67 
Table 2 Treatment characteristics of 81 recurrence and metastasis breast cancer patients

\begin{tabular}{lc}
\hline Treatment characteristics & Cases, $\mathrm{n}(\%)$ \\
\hline Adjuvant chemotherapy & $15(18.5)$ \\
Anthracyclines & $3(3.7)$ \\
Taxanes & $36(44.4)$ \\
Anthracyclines + taxanes & $6(7.4)$ \\
Unknown & \\
Adjuvant endocrine therapy & $43(53.1)$ \\
SERM & $20(24.7)$ \\
Al & \\
Adjuvant radiotherapy & $40(49.4)$ \\
No & $31(38.3)$ \\
Yes & \\
DFS (months) & $15(18(18.5)$ \\
$<24.0$ & $57(70.4)$ \\
$\geq 24.0$ & \\
Endocrine therapy sensitivity & \\
High & \\
Moderate & \\
Low & \\
CDK4/6i treatment line & \\
\hline
\end{tabular}

SERM, selective estrogen receptor modulators; Al, aromatase inhibitors; DFS, Disease-free survival.

and PR in primary tissue were available in 54 patients, and 74 cases, respectively (Table 1). In metastatic tissue, $77.8 \%$ $(\mathrm{n}=63)$ patients had Ki67 $\geq 14 \%, 45.7 \%(\mathrm{n}=37)$ had Ki67 $\geq 30 \%$, and $29.6 \%(\mathrm{n}=24)$ had PR $\geq 20 \%$ (Table 1 ).

In the total population, patients with Ki67 $\geq 14 \%$ had marginally significant shorter PFS than those with Ki67 <14\% [6.0 vs. 10.8 months; hazard ratio (HR) 1.94; 95\% confidence interval (CI): 0.95-3.96; $\mathrm{P}=0.062$; Figure $1 A$ ]. Patients with Ki67 $\geq 30 \%$ had significantly shorter PFS than those with Ki67 <30\% (6.0 vs. 8.5 months; HR 1.68; 95\% CI: 1.0-2.81; P=0.048; Figure $1 B$ ). The change in Ki67 from primary tissue to metastatic lesions was related to PFS, which showed that patients with Ki67 remaining at a low level or who changed from high to low had longer PFS than those whose Ki67 remained at a high level or who changed from low to high (Figure 1C). Furthermore, $\mathrm{PR} \geq 20 \%$ was associated with longer PFS (8.5 vs. 6.7 months; HR 0.59; 95\% CI: 0.32-1.08; $\mathrm{P}=0.08$; Figure 1D). Meanwhile, the change of PR from primary tissue to metastatic lesions was related to PFS, which showed that patients with PR remained at a high level or who changed from low to high had longer PFS than those whose PR remained at a low level or who changed from high to low (Figure 1E).

In regard to the hormone therapy subgroup, Ki67 and PR levels did not impact the PFS in the cohort treated with the fulvestrant in combination with palbociclib. However, there were significant associations between Ki67 and PR index and PFS in the AI subgroup. Patients with Ki67 $\geq 14 \%$ had shorter PFS than those with Ki67 $<14 \%$ (5.8 months vs. not reach; HR 3.69; 95\% CI: 1.1-12.42; $\mathrm{P}=0.024$; Figure $2 A$ ). Patients with Ki67 $\geq 30 \%$ had a significantly shorter PFS than those with Ki67 <30\% (5.1 vs. 11.8 months; HR 4.68; 95\% CI: 2.01-10.9; $\mathrm{P}<0.001$; Figure $2 B)$. Additionally, the change in Ki67 from primary tissue to metastatic lesions was related to PFS, which showed that patients whose Ki67 remained at a low level or who changed from high to low had longer PFS than those with Ki67 remaining at a high level or who changed from low to high (Figure 2C). PR $\geq 20 \%$ was associated with longer PFS (5.8 months $v$ s. not reach; HR 0.59; 95\% CI: 0.32-1.08; $\mathrm{P}=0.012$; Figure 2D). Noticeably, change in PR from primary tissue to metastatic lesions was related to PFS, which showed that patients with PR remaining at a high level or who changed from low to high had longer PFS than those with PR remaining at a low level or who changed from high to low (Figure 2E).

When both Ki67 and PR were considered, there were significant differences between the cohorts. Compared with patients who had $\mathrm{Ki} 67 \geq 14 \%$ and $\mathrm{PR}<20 \%$, those with Ki67 $<14 \%$ and PR $\geq 20 \%$ had significantly longer PFS (Figure 3A). In addition, patients with Ki67 $<30 \%$ and PR $\geq 20 \%$ had significantly longer PFS than those with Ki67 $\geq 30 \%$ and PR $<20 \%$ (Figure 3B). Finally, in the AI cohort, patients with Ki67 $<14 \%$ and PR $\geq 20 \%$ had significantly longer PFS than those with Ki67 $\geq 14 \%$ and PR $<20 \%$ (Figure 3C). Women with Ki67 $<30 \%$ and PR $\geq 20 \%$ had 
A

Progression-free survival

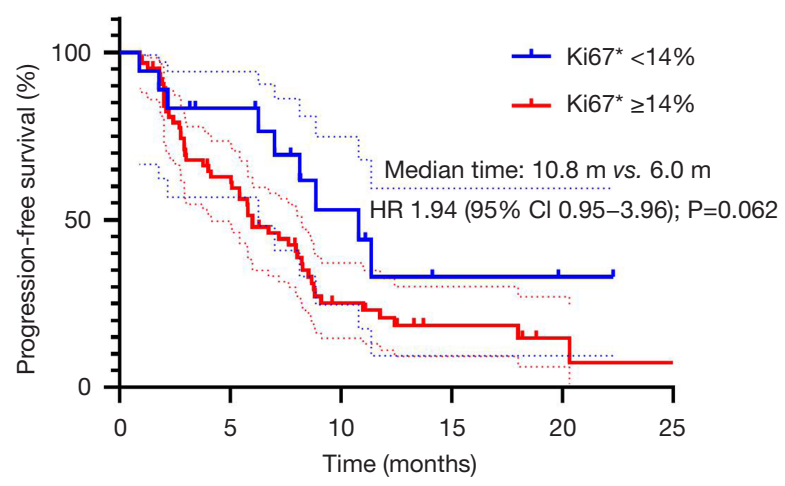

C

Progression-free survival

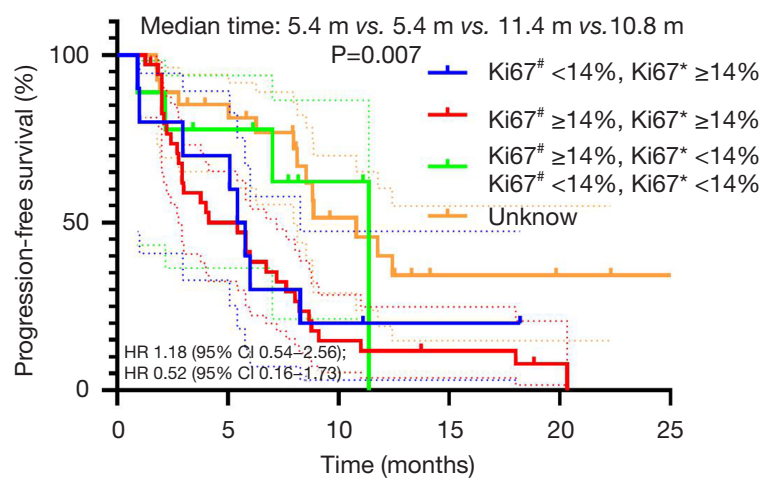

$E$

Progression-free survival

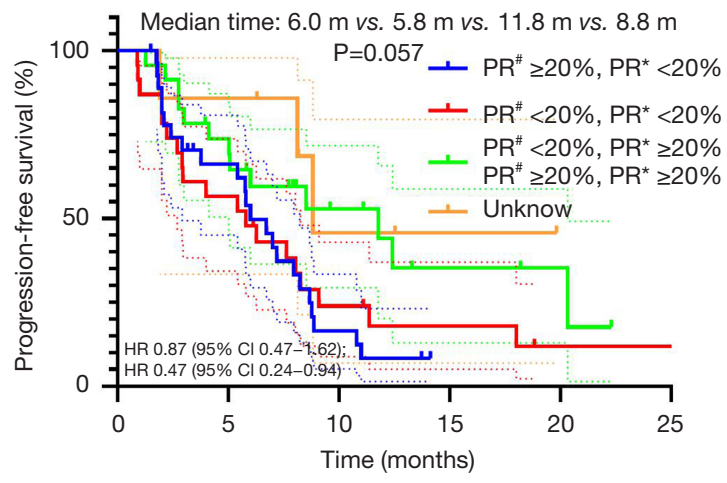

B Progression-free survival

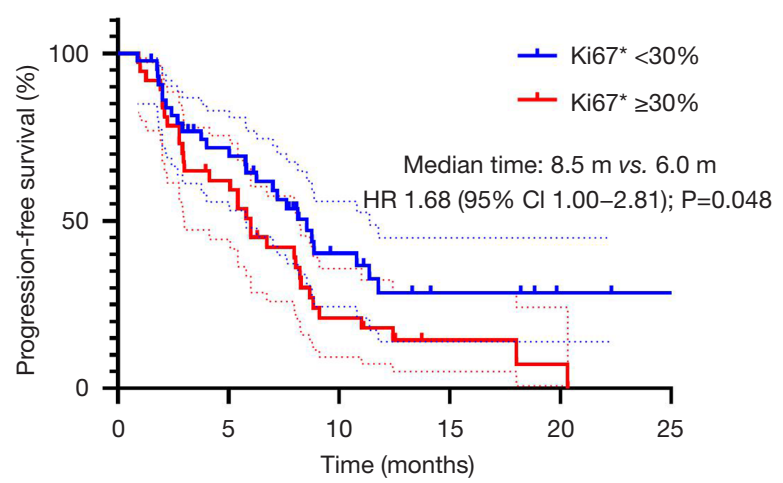

D

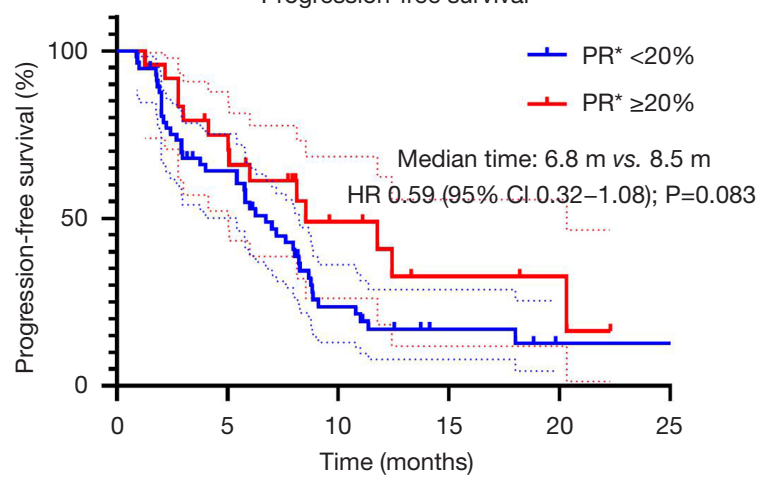

Figure 1 Progression-free survival in the total population. (A) Progression-free survival according to Ki $67<14 \%$ or $\geq 14 \%$; (B) progressionfree survival according to Ki67 $<30 \%$ or $\geq 30 \%$; (C) progression-free survival according to the change of Ki67 expression between primary tissue and metastatic lesions; (D) progression-free survival according to $\mathrm{PR}<20 \%$ or $\geq 20 \%$; (E) progression-free survival according to the change of PR expression between primary tissue and metastatic lesions. ", primary tumor; *, metastases. PR, progesterone receptor. 
A

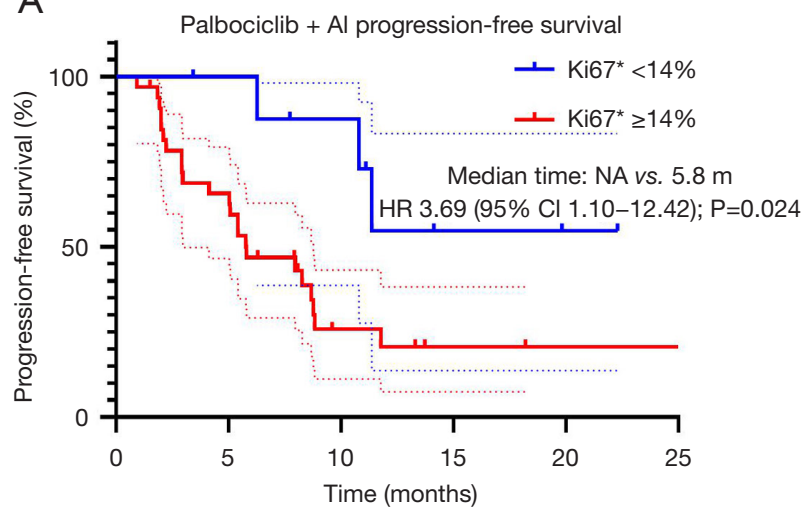

C Palbociclib + Al progression-free survival
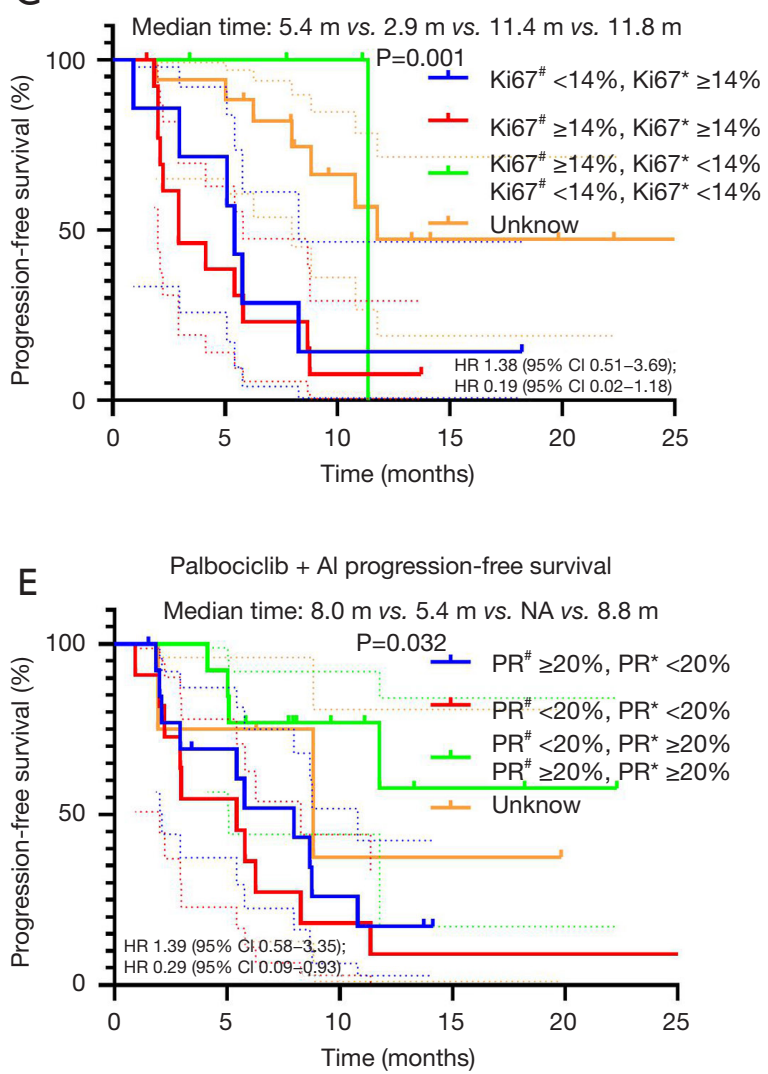

B
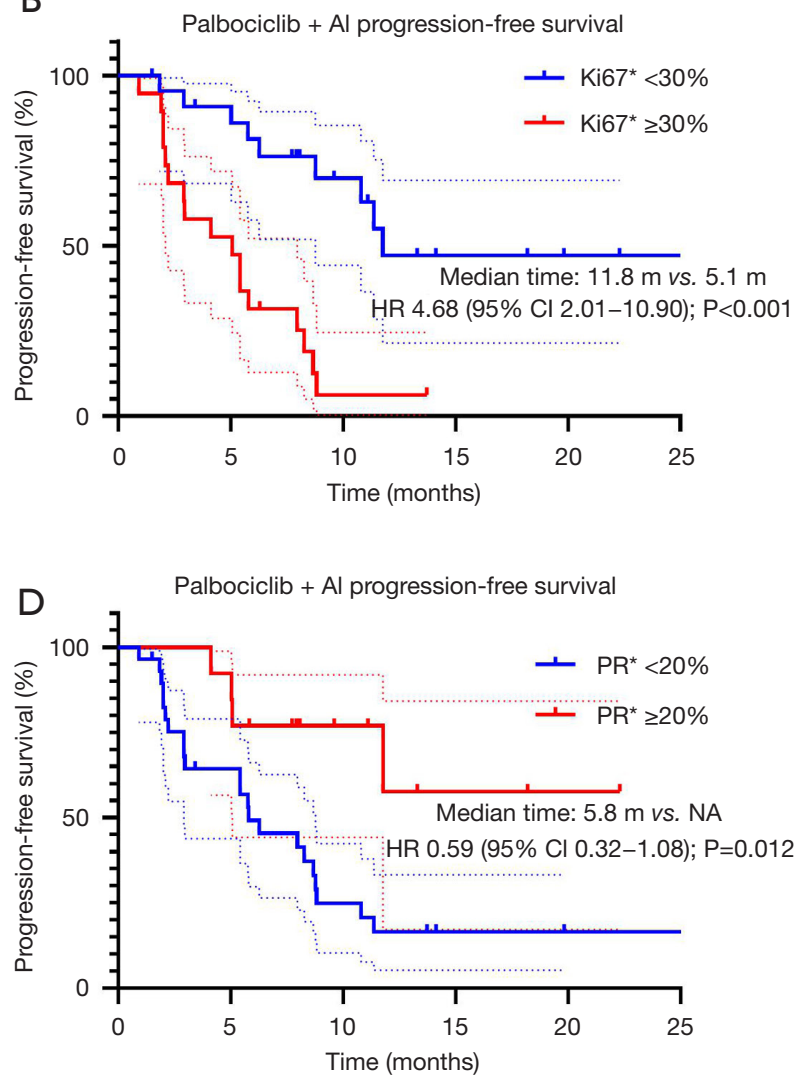

Figure 2 Progression-free survival in palbociclib combination with AI cohort. (A) Progression-free survival according to Ki67<14\% or $\geq 14 \%$; (B) progression-free survival according to Ki67 $<30 \%$ or $\geq 30 \%$; (C) progression-free survival according to the change of Ki67 expression between primary tissue and metastatic lesions; (D) progression-free survival according to PR $<20 \%$ or $\geq 20 \%$; (E) progressionfree survival according to the change of PR expression between primary tissue and metastatic lesions. *, primary tumor; *, metastases. AI, aromatase inhibitors; PR, progesterone receptor. 


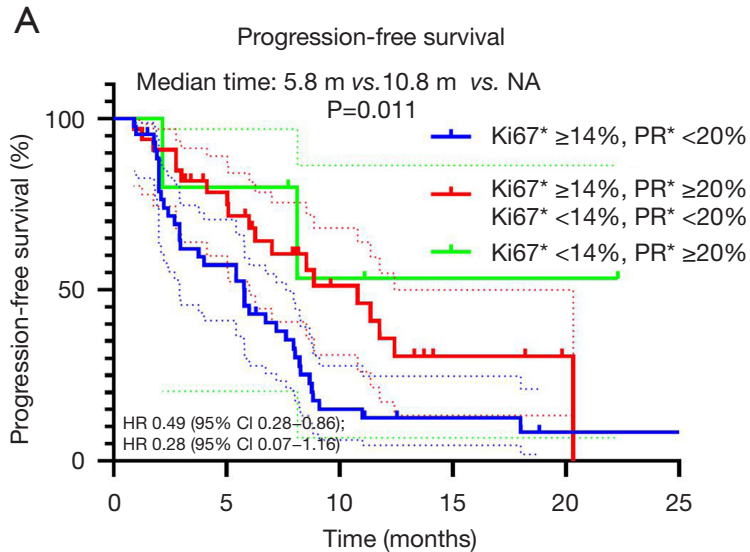

C

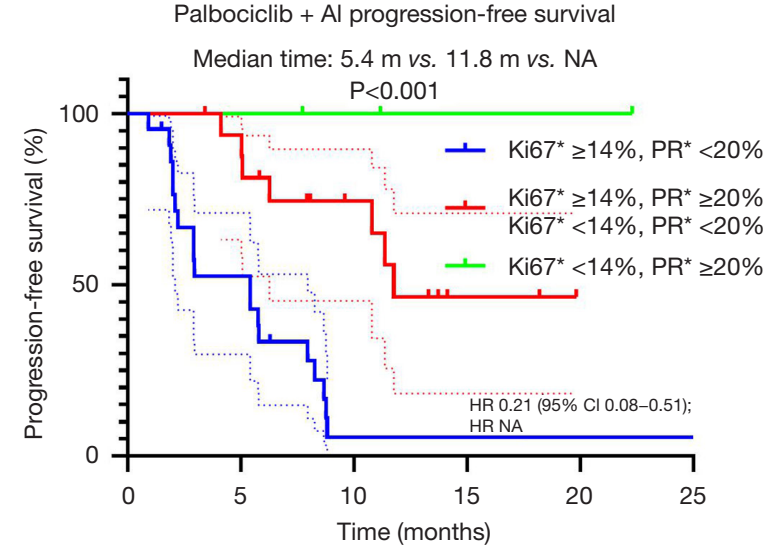

B

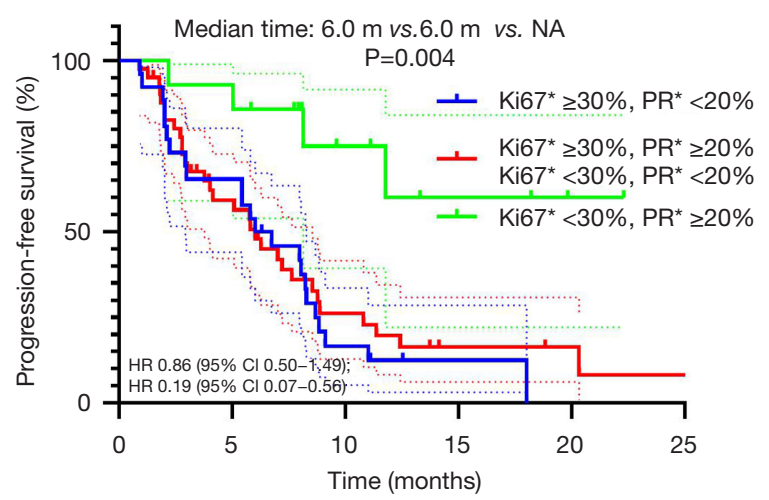

D

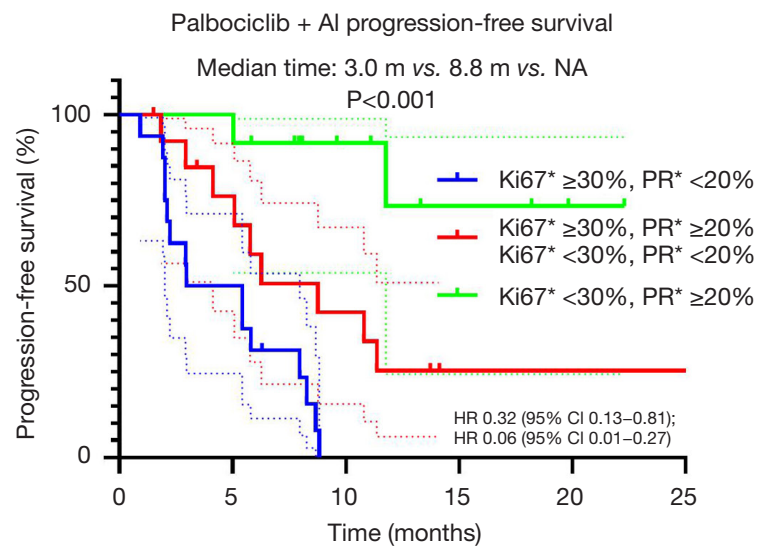

Figure 3 Progression-free survival in according to Ki67 and PR values. (A) Progression-free survival according to Ki67<14\% or $\geq 14 \%$ and $\mathrm{PR}<20 \%$ or $\geq 20 \%$ in the total population; (B) progression-free survival according to Ki67<30\% or $\geq 30 \%$ and $\mathrm{PR}<20 \%$ or $\geq 20 \%$ in the total population; (C) progression-free survival according to Ki67<14\% or $\geq 14 \%$ and $\mathrm{PR}<20 \%$ or $\geq 20 \%$ in palbociclib combination with AI cohort; (D) progression-free survival according to Ki67<30\% or $\geq 30 \%$ and $\mathrm{PR}<20 \%$ or $\geq 20 \%$ in palbociclib combination with $\mathrm{AI}$ cohort. ", primary tumor; *, metastases. PR, progesterone receptor; AI, aromatase inhibitors.

significantly longer PFS than those with Ki67 $\geq 30 \%$ and PR $<20 \%$ (Figure 3D).

\section{Discussion}

Palbociclib has demonstrated excellent clinical outcome in the data from the United States and Europe in the realworld setting with various treatment patterns and patient characteristics (27-31). In addition, the clinical benefit rates of palbociclib plus letrozole or fulvestrant demonstrated in the Ibrance Real World Insights (IRIS) study were all more than $90 \%$, regardless of the treatment line, which were better than the results of the PALOMA studies $85 \%$ in
PALOMA-2 and 67\% in PALOMA-3) (28,30). In addition, Asian patients were shown to receive greater benefit in PFS from CDK4/6i and endocrine therapy (32). Although these results are promising, the clinical practice of CDK $4 / 6$ inhibitors is confounded by the high individual variability in clinical response. At the current time, no clinically available biomarkers, other than ER expression, which is mostly derived from primary tissue, are used to prescribe CDK4/6 inhibitors (33-37). In addition, the ER-PR discordance between primary tumor and metastatic lesions is common and may reach approximately $32 \%$, leading to changes in treatment sensitivity (38). The present study represents the first retrospective real-world analysis demonstrating the 
correlation between Ki67and PR values of metastatic lesions and PFS in ABC patients with palbociclib plus hormone therapy.

A large-pooled America Food and Drug Administration (FDA) group analysis showed that ER positivity was the best predictor of CDK4/6i and hormone therapy (19). However, $\mathrm{PR}$ status was not suggested as a predictive biomarker in the same pooled study (19). Another exploratory analysis revealed that tumor grade, PR expression, performance status, liver metastases, bone-only disease, disease-free interval from completion of adjuvant hormone therapy, and duration from first diagnosis to relapse had a prognostic significance. The research further indicted that prognostic biomarkers were significantly associated with receiving the greatest benefit from the combination of another CDK4/6i (39).

A great many of prospective and retrospective studies have demonstrated a compatible correlation between elevated Ki67 levels and inferior benefit from chemotherapy in breast cancer. For neoadjuvant therapy, numerous trials have suggested a significant relationship between raised Ki67 values and outcome for chemotherapy, as assessed by pathological and clinical response (40). In addition, several neoadjuvant hormone therapy studies have demonstrated that treatment-induced conversion in Ki67 could predict response and clinical outcome, even with short-term treatment $(41,42)$. The monarchE trial presented in the 2020 San Antonio Breast Cancer Symposium (SABCS) showed that in the abemaciclib combined with adjuvant endocrine therapy setting, patients with high Ki67 achieved a statistically significant improvement in invasive diseasefree survival (iDFS). However, little research has been carried out concerning the ability of Ki67 to predict the clinical outcome for hormone therapy or chemotherapy in a metastatic setting. Meanwhile, Ki67 value was not indicated to be a prognostic factor for response to CDK4/6i in a series of prospective trials. Only 1 retrospective study reported that PFS was negatively affected by elevated Ki67 values (22). The key challenge in Ki67 research is the poor interlaboratory assay reproducibility due to the diversity in diagnosis kits and platforms being used. Moreover, various cutoffs and score analyses have been applied throughout years of research $(40,43)$. In a metastatic setting, increased PR was shown to be independently associated with a long time-to-progression (TTP) in patients with hormone therapy, whereas Ki67 was not (44). In addition to this, the PALOMA3 study indicated that high PR level is correlated with superior outcomes in patients with either placebo and fulvestrant or palbociclib combined with fulvestrant, independent of the treatment cohort (20). Ki67 has also been reported to be related to phosphorylated retinoblastoma protein $(\mathrm{Rb})$ in $\mathrm{Rb}$-proficient breast cancer (BC), with Rb-negative BC being associated with increased $\mathrm{Ki} 67$ values (21). Consequently, these Rb-deficient $\mathrm{BC}$ cases with high proliferative activity are speculated to be resistant to CDK4/6i (45).

We investigated the impact of Ki67 and $\mathrm{PR}$ values on CDK4/6i treatment in $\mathrm{HR}+$ /HER2 - ABC patients. A particular novelty of our study was the evaluation of the change in Ki67 and PR from primary tissue to metastatic lesions. Our study revealed that PFS seemed to be negatively influenced by elevated Ki67 values and low PR expression. Moreover, the change in Ki67 from primary tissue to metastatic lesions was also related to PFS. Patients with Ki67 remaining at a low level or who changed from high to low had longer PFS than those with Ki67 remaining at a high level or who changed from low to high. The change in PR from primary tissue to metastatic lesions was related to PFS, which showed that patients with PR remaining at a high level or who changed from low to high had longer PFS than those with PR remaining at a low level or who changed from high to low.

Palbociclib plus fulvestrant demonstrated the same clinical outcome as palbociclib in combination with AI, which was consistent with the data from the PARSIFAL trial presented in the 2020 ASCO (46). Also, there was no difference in clinical outcome between these 2 subgroups in the present study. Of further note, Ki67 and PR levels had no impact on the clinical outcome of palbociclib plus fulvestrant, but greatly affected the benefit of palbociclib plus AIs. Women with high Ki67 expression or low PR expression received less benefit from palbociclib plus AIs, but may still be sensitive to palbociclib and fulvestrant. Meanwhile, patients with Ki67 remaining at a low level or who changed from high to low had longer PFS than those with Ki67 remaining at a high level or who changed from low to high. Those patients with PR remaining at a high level or who changed from low to high had longer PFS than those with PR remaining at a low level or who changed from high to low. These findings may provide insight into the optimal selection of palbociclib partners using Ki67 and PR values.

In addition, 10 patients underwent second-generation sequencing (NGS). This reveal that 7 patients with PIK3CA mutation had a PFS of only 3.0 months which was markedly shorter than that of the whole cohort which was 8.0 months. 


\section{Page 10 of 12}

Two of the patients who progressed from AI treatment had an ESR1 mutation and received palbociclib plus fulvestrant, as ESR1 mutation is one of the main causes of AI resistance (47).

Despite its retrospective nature and the lack of a control cohort, our data reflect a real-world patient cohort which greatly differs from the populations included in most clinical trials. As Ki67 and PR detection is easily available, economical, and practical, the predictive role of Ki67 and PR on PFS in palbociclib treatment may be widely applicable and their value merits further confirmation in a prospective study.

\section{Acknowledgments}

Funding: This work was supported by grants from Zhejiang Provincial Natural Science Foundation (No. Y2101312 and LY17H160041), the Zhejiang Provincial Medical Science and Technology Program (No. 2010QNA006 and 2021KY551), Zhejiang science and Technology Program (No. 2016C33199), the Special Fund of Wu Jieping Medical Foundation Clinical Research (No. 320.670010007).

\section{Footnote}

Reporting Checklist: The authors have completed the REMARK reporting checklist. Available at http://dx.doi. org/10.21037/atm-21-1340

Data Sharing Statement: Available at http://dx.doi. org/10.21037/atm-21-1340

Conflicts of Interest: All authors have completed the ICMJE uniform disclosure form (available at http://dx.doi. org/10.21037/atm-21-1340). The authors have no conflicts of interest to declare.

Ethical Statement: The authors are accountable for all aspects of the work in ensuring that questions related to the accuracy or integrity of any part of the work are appropriately investigated and resolved. This retrospective study was conducted in line with accepted ethical standards and was approved by the Medical Scientific Committee of Cancer Hospital of the University of Chinese Academy of Sciences/Zhejiang Cancer Hospital (No. IRB-2021-19). All procedures performed in this study involving human participants were in accordance with the Declaration of Helsinki (as revised in 2013). Individual consent for this
Shao et al. Ki67 and PR status predicts sensitivity to palbociclib

retrospective analysis was waived.

Open Access Statement: This is an Open Access article distributed in accordance with the Creative Commons Attribution-NonCommercial-NoDerivs 4.0 International License (CC BY-NC-ND 4.0), which permits the noncommercial replication and distribution of the article with the strict proviso that no changes or edits are made and the original work is properly cited (including links to both the formal publication through the relevant DOI and the license). See: https://creativecommons.org/licenses/by-nc-nd/4.0/.

\section{References}

1. Cardoso F, Costa A, Norton L, et al. ESO-ESMO 2nd international consensus guidelines for advanced breast cancer (ABC2) dagger. Ann Oncol 2014;25:1871-88.

2. Turner NC, Neven P, Loibl S, et al. Advances in the treatment of advanced oestrogen-receptor-positive breast cancer. Lancet 2017;389:2403-14.

3. Matikas A, Foukakis T, Bergh J. Tackling endocrine resistance in ER-positive HER2-negative advanced breast cancer: A tale of imprecision medicine. Crit Rev Oncol Hematol 2017;114:91-101.

4. Ellis M. Overcoming endocrine therapy resistance by signal transduction inhibition. Oncologist 2004;9 Suppl 3:20-6.

5. Finn RS, Crown JP, Lang I, et al. The cyclin-dependent kinase 4/6 inhibitor palbociclib in combination with letrozole versus letrozole alone as first-line treatment of oestrogen receptor-positive, HER2-negative, advanced breast cancer (PALOMA-1/TRIO-18): a randomised phase 2 study. Lancet Oncol 2015;16:25-35.

6. Beaver JA, Amiri-Kordestani L, Charlab R, et al. FDA Approval: Palbociclib for the Treatment of Postmenopausal Patients with Estrogen Receptor-Positive, HER2-Negative Metastatic Breast Cancer. Clin Cancer Res 2015;21:4760-6.

7. Spring LM, Wander SA, Zangardi M, et al. CDK 4/6 Inhibitors in Breast Cancer: Current Controversies and Future Directions. Curr Oncol Rep 2019;21:25.

8. Ballinger TJ, Meier JB, Jansen VM. Current Landscape of Targeted Therapies for Hormone-Receptor Positive, HER2 Negative Metastatic Breast Cancer. Front Oncol 2018;8:308.

9. Hortobagyi GN, Stemmer SM, Burris HA, et al. Ribociclib as First-Line Therapy for HR-Positive, Advanced Breast Cancer. N Engl J Med 2016;375:1738-48. 
10. Goetz MP, Toi M, Campone M, et al. MONARCH 3: Abemaciclib As Initial Therapy for Advanced Breast Cancer. J Clin Oncol 2017;35:3638-46.

11. Finn RS, Martin M, Rugo HS, et al. Palbociclib and Letrozole in Advanced Breast Cancer. N Engl J Med 2016;375:1925-36.

12. Turner NC, Ro J, Andre F, et al. Palbociclib in HormoneReceptor-Positive Advanced Breast Cancer. N Engl J Med 2015;373:209-19.

13. Sledge GW Jr, Toi M, Neven P, et al. MONARCH 2: Abemaciclib in Combination With Fulvestrant in Women With HR+/HER2- Advanced Breast Cancer Who Had Progressed While Receiving Endocrine Therapy. J Clin Oncol 2017;35:2875-84.

14. Slamon DJ, Neven P, Chia S, et al. Phase III Randomized Study of Ribociclib and Fulvestrant in Hormone ReceptorPositive, Human Epidermal Growth Factor Receptor 2-Negative Advanced Breast Cancer: MONALEESA-3.J Clin Oncol 2018;36:2465-72.

15. Cristofanilli M, Turner NC, Bondarenko I, et al. Fulvestrant plus palbociclib versus fulvestrant plus placebo for treatment of hormone-receptor-positive, HER2negative metastatic breast cancer that progressed on previous endocrine therapy (PALOMA-3): final analysis of the multicentre, double-blind, phase 3 randomised controlled trial. Lancet Oncol 2016;17:425-39.

16. Collins R, Bowman L, Landray M, et al. The Magic of Randomization versus the Myth of Real-World Evidence. N Engl J Med 2020;382:674-8.

17. Hoste G, Punie K, Wildiers H, et al. Palbociclib in highly pretreated metastatic ER-positive HER2-negative breast cancer. Breast Cancer Res Treat 2018;171:131-41.

18. Ban M, Mise BP, Majic A, et al. Efficacy and safety of palbociclib in heavily pretreated patients with HR+/HER2metastatic breast cancer. Future Oncol 2018;14:537-44.

19. Gao JJ, Cheng J, Bloomquist E, et al. CDK4/6 inhibitor treatment for patients with hormone receptor-positive, HER2-negative, advanced or metastatic breast cancer: a US Food and Drug Administration pooled analysis. Lancet Oncol 2020;21:250-60.

20. Cristofanilli M, DeMichele A, Giorgetti C, et al. Predictors of prolonged benefit from palbociclib plus fulvestrant in women with endocrine-resistant hormone receptor-positive/human epidermal growth factor receptor 2-negative metastatic breast cancer in PALOMA-3. Eur J Cancer 2018;104:21-31.

21. Ceccarelli C, Santini D, Chieco P, et al. Retinoblastoma (RB1) gene product expression in breast carcinoma.
Correlation with Ki-67 growth fraction and biopathological profile. J Clin Pathol 1998;51:818-24.

22. Palleschi M, Maltoni R, Ravaioli S, et al. Ki67 and PR in Patients Treated with CDK4/6 Inhibitors: A Real-World Experience. Diagnostics (Basel) 2020;10:573.

23. Wolff AC, Hammond ME, Hicks DG, et al. Recommendations for human epidermal growth factor receptor 2 testing in breast cancer: American Society of Clinical Oncology/College of American Pathologists clinical practice guideline update. J Clin Oncol 2013;31:3997-4013.

24. Hammond ME, Hayes DF, Wolff AC. Clinical Notice for American Society of Clinical Oncology-College of American Pathologists guideline recommendations on ER/PgR and HER2 testing in breast cancer. J Clin Oncol 2011;29:e458.

25. Goldhirsch A, Winer EP, Coates AS, et al. Personalizing the treatment of women with early breast cancer: highlights of the St Gallen International Expert Consensus on the Primary Therapy of Early Breast Cancer 2013. Ann Oncol 2013;24:2206-23.

26. Schemper M, Smith TL. A note on quantifying followup in studies of failure time. Control Clin Trials 1996;17:343-6.

27. Pizzuti L, Giordano A, Michelotti A, et al. Palbociclib plus endocrine therapy in HER2 negative, hormonal receptorpositive, advanced breast cancer: A real-world experience. J Cell Physiol 2019;234:7708-17.

28. Taylor-Stokes G, Mitra D, Waller J, et al. Treatment patterns and clinical outcomes among patients receiving palbociclib in combination with an aromatase inhibitor or fulvestrant for HR+/HER2-negative advanced/metastatic breast cancer in real-world settings in the US: Results from the IRIS study. Breast 2019;43:22-7.

29. Varella L, Eziokwu AS, Jia X, et al. Real-world clinical outcomes and toxicity in metastatic breast cancer patients treated with palbociclib and endocrine therapy. Breast Cancer Res Treat 2019;176:429-34.

30. Waller J, Mitra D, Mycock K, et al. Real-World Treatment Patterns and Clinical Outcomes in Patients Receiving Palbociclib for Hormone Receptor-Positive, Human Epidermal Growth Factor Receptor 2-Negative Advanced or Metastatic Breast Cancer in Argentina: The IRIS Study. J Glob Oncol 2019;5:JGO1800239.

31. Wilkie J, Schickli MA, Berger MJ, et al. Progression-Free Survival for Real-World Use of Palbociclib in Hormone Receptor-Positive Metastatic Breast Cancer. Clin Breast Cancer 2020;20:33-40. 


\section{Page 12 of 12}

32. Ramos-Esquivel A, Hernandez-Steller H, Savard MF, et al. Cyclin-dependent kinase 4/6 inhibitors as firstline treatment for post-menopausal metastatic hormone receptor-positive breast cancer patients: a systematic review and meta-analysis of phase III randomized clinical trials. Breast Cancer 2018;25:479-88.

33. Fang H, Huang D, Yang F, et al. Potential biomarkers of CDK4/6 inhibitors in hormone receptor-positive advanced breast cancer. Breast Cancer Res Treat 2018;168:287-97.

34. Guarducci C, Bonechi M, Boccalini G, et al. Mechanisms of Resistance to CDK4/6 Inhibitors in Breast Cancer and Potential Biomarkers of Response. Breast Care (Basel) 2017;12:304-8.

35. Pandey K, An HJ, Kim SK, et al. Molecular mechanisms of resistance to CDK4/6 inhibitors in breast cancer: A review. Int J Cancer 2019;145:1179-88.

36. Portman N, Alexandrou S, Carson E, et al. Overcoming CDK4/6 inhibitor resistance in ER-positive breast cancer. Endocr Relat Cancer 2019;26:R15-30.

37. Garrido-Castro AC, Goel S. CDK4/6 Inhibition in Breast Cancer: Mechanisms of Response and Treatment Failure. Curr Breast Cancer Rep 2017;9:26-33.

38. Sperduto PW, Mesko S, Li J, et al. Estrogen/progesterone receptor and HER2 discordance between primary tumor and brain metastases in breast cancer and its effect on treatment and survival. Neuro Oncol 2020;22:1359-67.

39. Di Leo A, O'Shaughnessy J, Sledge GW Jr, et al. Prognostic characteristics in hormone receptor-positive advanced breast cancer and characterization of abemaciclib efficacy. NPJ Breast Cancer 2018;4:41.

40. Dowsett M, Nielsen TO, A'Hern R, et al. Assessment of Ki67 in breast cancer: recommendations from the International Ki67 in Breast Cancer working group. J Natl Cancer Inst 2011;103:1656-64.

Cite this article as: Shao X, Zheng Y, Cao W, Shen X, Li G, Chen J, Huang Y, Huang P, Shi L, Ye W, Zou W, Lou C, Lei L, Huang J, Chen Z, Wang X. Ki67 and progesterone receptor status predicts sensitivity to palbociclib: a real-world study. Ann Transl Med 2021;9(8):707. doi: 10.21037/atm-21-1340
Shao et al. Ki67 and PR status predicts sensitivity to palbociclib

41. Tanei T, Shimomura A, Shimazu K, et al. Prognostic significance of Ki67 index after neoadjuvant chemotherapy in breast cancer. Eur J Surg Oncol 2011;37:155-61.

42. Jones RL, Salter J, A'Hern R, et al. The prognostic significance of Ki67 before and after neoadjuvant chemotherapy in breast cancer. Breast Cancer Res Treat 2009;116:53-68.

43. Nielsen TO, Leung SCY, Rimm DL, et al. Assessment of Ki67 in Breast Cancer: Updated Recommendations from the International Ki67 in Breast Cancer Working Group. J Natl Cancer Inst 2020. [Epub ahead of print]. doi: 10.1093/jnci/djaa201.

44. Rocca A, Farolfi A, Maltoni R, et al. Efficacy of endocrine therapy in relation to progesterone receptor and Ki67 expression in advanced breast cancer. Breast Cancer Res Treat 2015;152:57-65.

45. Finn RS, Dering J, Conklin D, et al. PD 0332991, a selective cyclin D kinase 4/6 inhibitor, preferentially inhibits proliferation of luminal estrogen receptor-positive human breast cancer cell lines in vitro. Breast Cancer Res 2009;11:R77.

46. Llombart-Cussac A, Perez-Garcia JM, Bellet M, et al. PARSIFAL: A randomized, multicenter, open-label, phase II trial to evaluate palbociclib in combination with fulvestrant or letrozole in endocrine-sensitive patients with estrogen receptor (ER)[+]/HER2 [-] metastatic breast cancer. J Clin Oncol 2020;38:Suppl:1007-1007.

47. Turner NC, Swift C, Kilburn L, et al. ESR1 Mutations and Overall Survival on Fulvestrant versus Exemestane in Advanced Hormone Receptor-Positive Breast Cancer: A Combined Analysis of the Phase III SoFEA and EFECT Trials. Clin Cancer Res 2020;26:5172-7. 